\title{
Nueva realidad: compras en línea y a domicilio
}

\author{
fecha de recepción: 2020-07-25 • Fecha de aceptación: 2020-08-20 • Fecha de publicación: 2020-10-10
}

\author{
Andrea Jenniffer Reyes Rivera ${ }^{1}$ \\ Quest, Ecuador \\ andyjrey@gmail.com \\ https://orcid.org/0000-0002-3495-5484 \\ María de Lourdes Vallejos Cango ${ }^{2}$ \\ Consejo de la Judicatura, Ecuador \\ mary.lu126117@gmail.com \\ https://orcid.org/0000-0003-1035-0699 \\ Daniel Alonso Quintana García ${ }^{3}$ \\ Nexsys Del Ecuador, Ecuador \\ danielquintana.ag@gmail.com \\ https://orcid.org/0000-0003-3637-4963
}

\section{RESUMEN}

A propósito de los temas coyunturales a nivel mundial, se ha visto un aumento en el uso de las plataformas digitales en todos los sectores, desde el educativo, comercial o gubernamental, ya sea para realizar una videoconferencia o para hacer una compra o venta de un producto. Este trabajo investigativo describe la creación y funcionamiento de una aplicación que satisfaga estas necesidades actuales del mercado, y a futuro podría competir con aquellas de nivel extranjero. Como resultado del desarrollo se pone a disposición un pool de productos que podrán ser ingresados en un carrito de compras y posterior a su confirmación, localizar la dirección del cliente para su entrega a domicilio, esto debido a las medidas de emergencias por consecuencia del COVID-19, además de ser de mayor comodidad para el usuario. 


\section{ABSTRACT}

With regard to global issues, there has been an increase in the use of digital platforms in all sectors, from education, commercial or government, either to make a video conference or to make a purchase or sale of a product. This research describes the creation and operation of an application that meets these current market needs, and in the future could compete with those of a foreign level. As a result of the development, a pool of products is made available that can be put in a shopping cart and, after confirmation, locate the customer's address for home delivery, this due to the emergency measures as a result of the COVID-19, in addition to being more convenient for the user.

KEYWORDS: product, online shopping, platform, commercial, address, covid-19. 


\section{Introducción}

El desarrollo tecnológico y el acceso a grandes cantidades de información en la web han alcanzado un nivel sin precedentes en la historia (Dinu y Dinu, 2014), y es que el Internet se convierte en un medio potencial de distribución y su desarrollo está afectando claramente a los hábitos de consumo de todas las personas a nivel mundial (Caldito, 2005), y más ahora que se está viviendo una pandemia tan grave como lo es el CODIV-19.

Según Jason Droege, vicepresidente de UberEverything, desde que Uber nació en 2009, la pregunta obligada fue: ¿Cómo crecer más y llegar a más ciudades? "Llevo cuatro años en la compañía. Había Uber para flores, marketing...a través de mucha experimentación llegamos a la comida. Nos ha permitido crecer en muchos otros negocios a través de la plataforma" (Contreras, 2018).

Su equipo creó Uber Eats, la app que permite recibir entregas de comida en menos de 31 minutos en 350 ciudades de 36 países. Esta semana llega a Centroamérica, El Salvador, donde ya comenzó la búsqueda para encontrar socios repartidores de la plataforma. En cambio, Uber ofrece información acerca de horas y zonas con más actividad para acceder a más viajes, soporte 24/7 para resolver cualquier problema, participar en promociones y descuentos especiales. Uber Eats llega a competir con dos plataformas virtuales de reparto de comida a domicilio: Gourmet Express y Hugo App, la startup salvadoreña para entregas a domicilio (Contreras, 2018).

Droege explicó que cuando todo arrancó no era regular que muchos restaurantes ofrecieran el servicio de envío de comida. "Con eso en mente, y ya que la industria de restaurantes ha cambiado a más casual food, la gente está tomando take away más", agregó Droege, en referencia a la app que nació en 2015 (Contreras, 2018).

Por su parte, el fundador de Glovo, Óscar Pierre, afirma que "en el futuro la gente tendrá múltiples vías de ingresos", y defiende que su empresa aporta una flexibilidad muy valorada por los repartidores a domicilio. Prevé facturar 100 millones en 2018 y elevar esto en 2020 (Figuls, 2018).

Según la consultora especializada App Annie, uno de los primeros hitos tras la aparición del Covid-19 está relacionado con el incremento en las descargas y las horas dedicadas a las aplicaciones Android e IOS con foco en actividades vinculadas a la productividad y a la educación, de la misma manera, también se ha registrado un fuerte crecimiento en las descargas de juegos en los mercados afectados, ya que los consumidores recurren a los dispositivos móviles para entretenerse y pasar el tiempo en cuarentena, dado la alta penetración de los dispositivos con sistema operativo Android, comparado con los que tienen iOS (SLOTNISKY, 2020).

En este contexto, se propone el desarrollo de una plataforma que permite al vendedor comercializar sus productos a través del internet, esta plataforma está desarrollada con el lenguaje de programación JAVA y el IDE Android Studio, el modelo comercial que apalanca la plataforma es un modelo de negocio B2C (Li, F., \& Li, Y, 2011). 


\section{Metodología}

En la presente investigación la metodología que se ha usado para llevar a cabo la gestión de este proyecto es Scrum (Figura 1):

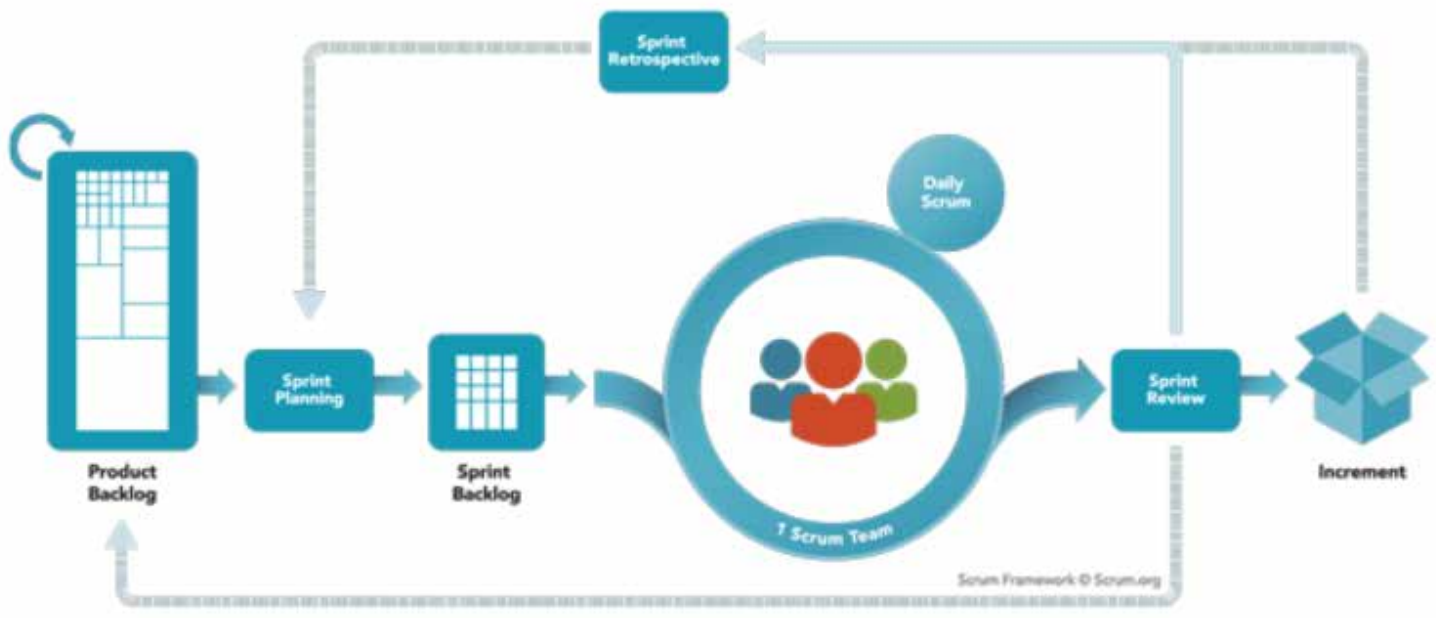

Download The Scrum Framework

Figura 1. Imagen ilustrativa Scrum

Fuente: Scrum.org (2020)

A su vez, se utilizó un equipo de trabajo (Tabla 1) que estuvo dividido de la siguiente manera:

Tabla 1.

Equipo de trabajo

\begin{tabular}{ll}
\hline Product Owner & Andrea Reyes \\
\hline Scrum Master & María de Lourdes Vallejos \\
\hline Equipo de Desarrollo & Andrea Reyes, Daniel Quintana \\
\hline
\end{tabular}

Fuente: elaboración propia

A continuación, en la Tabla 2 se detallan las historias de usuarios que fueron creados en la fase inicial del proyecto. 
Tabla 2.

Historia de usuario

\begin{tabular}{ll}
\hline & HISTORIA DE USUARIO \\
\hline Número: RF01 & Nombre(Rol): Clientes. \\
\hline Usuario: Usuario & Riesgo en Desarrollo: Alta \\
\hline Prioridad en negocio: Alta & \\
\hline
\end{tabular}

Descripción: Los usuarios deben estar registrados para poder ingresar al sistema.

- Se deberá registrar nombre, apellido.

- Se deberá registrar Email a través de la cuenta de Gmail.

- Se deberá ingresar Contraseña.

- Luego dar clic en el botón Regístrate.

- Se deben identificar con Usuario y Contraseña.

- Deberá dar un clic en el botón Login.

- Se validará los datos de autentificación con la cuenta de Google o los datos registrados en la base de datos.

- Permitirá el ingreso al aplicativo para la utilización del módulo de compras.

- $\quad$ El usuario deberá dar clic en el botón SELECCIONA TUS PRODUCTOS podrá seleccionar los productos.

- El usuario al dar clic en el botón PRODUCTOS EN EL CARRITO podrá visualizar la compra.

- $\quad$ El usuario al dar clic en el botón AGREGAR TU UBICACIÓN se llenará el campo de dirección.

- $\quad$ El usuario deberá dar check en el producto que escoja.

- $\quad$ El usuario deberá clic en el botón AGREGAR EN EL CARRITO.

- El usuario visualizará el valor de su pedido.

- El usuario deberá llenar los campos contacto y dirección

- El usuario deberá dar clic en el botón CONFIRMAR le enviará a otra pantalla solicitándole la ubicación.

- El usuario al dar clic en el icono propio de la APP

le llevará a la ubicación del usuario.

El usuario al dar clic en el botón ACEPTAR le mostrará un mensaje "Su compra fue confirmada y será enviada a la ubicación ingresada. Gracias por preferirnos".

Fuente: elaboración propia

Lo descrito anteriormente es un resumen de la metodología utilizada que permitió desarrollar el trabajo propuesto.

En cuanto al desarrollo de la app describiremos los elementos importantes que fueron necesarios en el proceso de creación.

\section{Base de datos SQLITE}

SQLite no requiere más que un simple fichero para almacenar los datos, ya que la lógica de funcionamiento debe ser implementada por la plataforma que desee interactuar con los datos. En nuestro caso concreto el SDK Android incluye soporte completo para SQLite (Kreibich J, 2010). 
La mayoría de las aplicaciones móviles, tanto en Android como en otros sistemas, incluyen bases de datos SQLite para la gestión total de los datos por medio de JSON.

\section{Objetos del API de Google}

En el desarrollo de esta aplicación fue fundamental el uso de API públicas como son la API de inicio de sesión y el API de geolocalizar y mapas, las cuales se referencian a continuación, por medio de su código fuente (Figura 2 y 3 ).

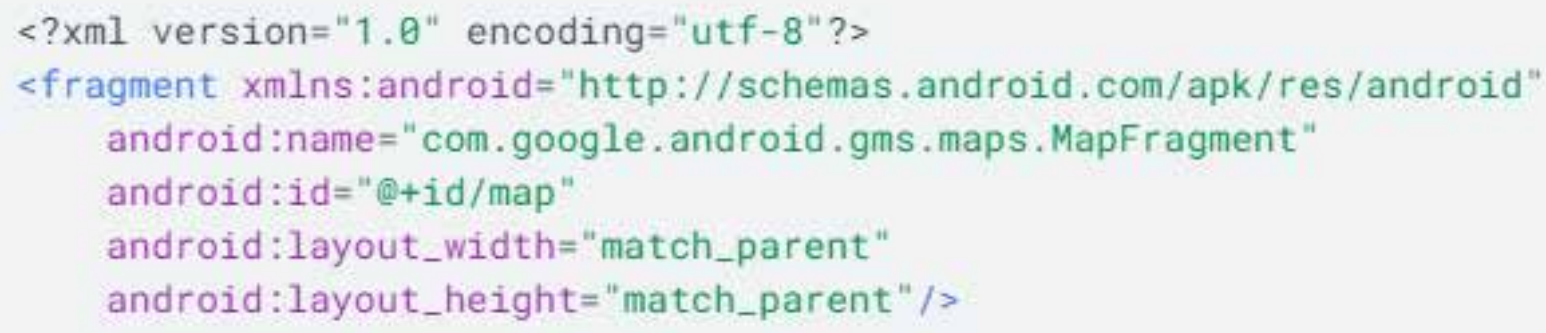

Figura 2. Fragmento de maps

Fuente: elaboración propia

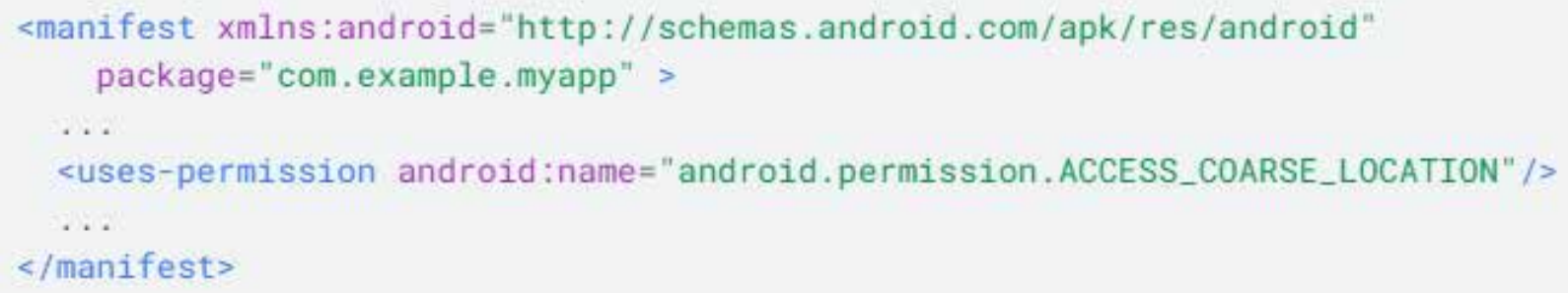

Figura 3. Datos de ubicación

Fuente: elaboración propia

En la siguiente Figura 4 se refleja el resultado de cómo se ve en el dispositivo móvil. 


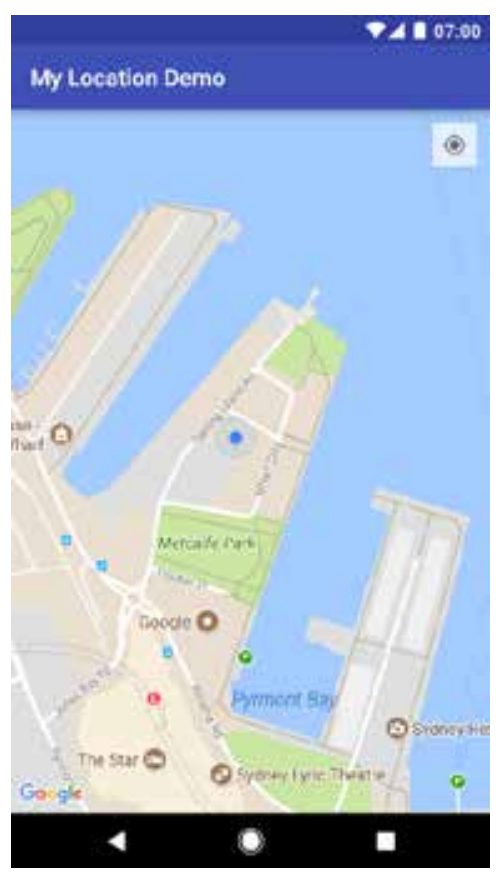

Figura 4. Ubicación en el mapa de dispositivo móvil

Fuente: elaboración propia

Luego se procedió a incluir la codificación para lo que sería el botón de inicio (Figura 5) de la aplicación.

\section{$<$ div class="g-signin2" data-onsuccess="onSignIn" $></$ div $>$}

Figura 5. Codificación para botón de inicio de sesión

Fuente: elaboración propia

En la Figura 6 se muestra cómo se podrá obtener información del perfil de un usuario.

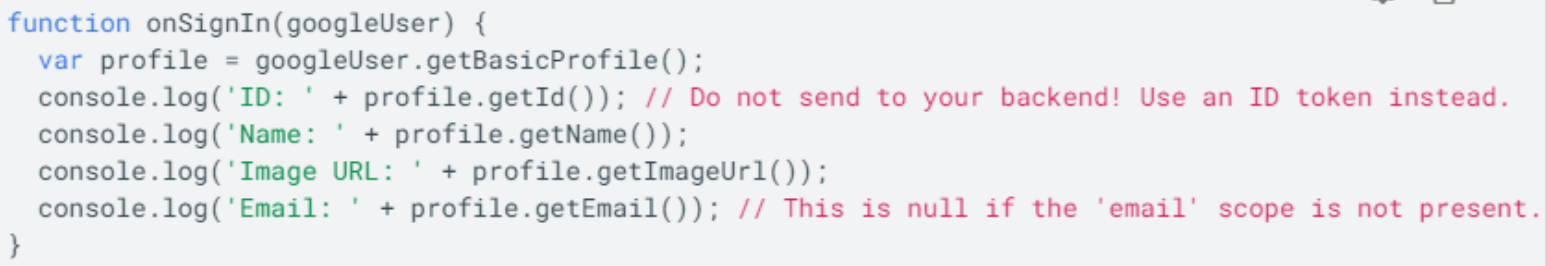

Figura 6. Información del perfil

Fuente: elaboración propia 
Y en la Figura 7 la codificación para cuando este cierre su sesión.

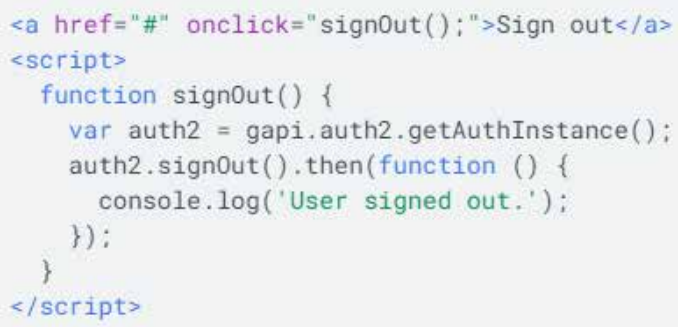

Figura 7. Cerrar sesión de un usuario

Fuente: elaboración propia

Se optó por colocar código fuente ya que facilita que algún otro proyecto pueda replicar la funcionalidad de este trabajo, debido a que en la documentación oficial de Android se detalla innumerables líneas de código, muchas veces es confuso por lo que se coloca únicamente el código necesario para cumplir con lo requerido.

\section{Resultados}

Esta sección describe de forma general el proceso que realiza un usuario al utilizar la App desarrollada (Figura 8) en este trabajo.

- Una vez realizado el registro local debe mostrar un mensaje como este: "Registrado Exitoso". (Figura 9)

- Como se muestra en la Figura 10, donde ingresa sus datos, en el caso de que la autentificación sea fallida debe mostrar un mensaje indicando "Usuario y/o contraseña incorrectos".

- El usuario deberá seguir los pasos (Figura 11) para poder realizar el pedido.

- Al hacer clic en AGREGAR EN EL CARRITO mostrará el pedido vacío. (Figura 12).

- En el caso de no llenar los campos de contacto y dirección mostrará el mensaje "Por favor ingrese los datos de contacto".

- Al dar clic en CONFIRMAR mostrará un mensaje así: "Permitir que RapiCompra acceda a la ubicación de este dispositivo". 


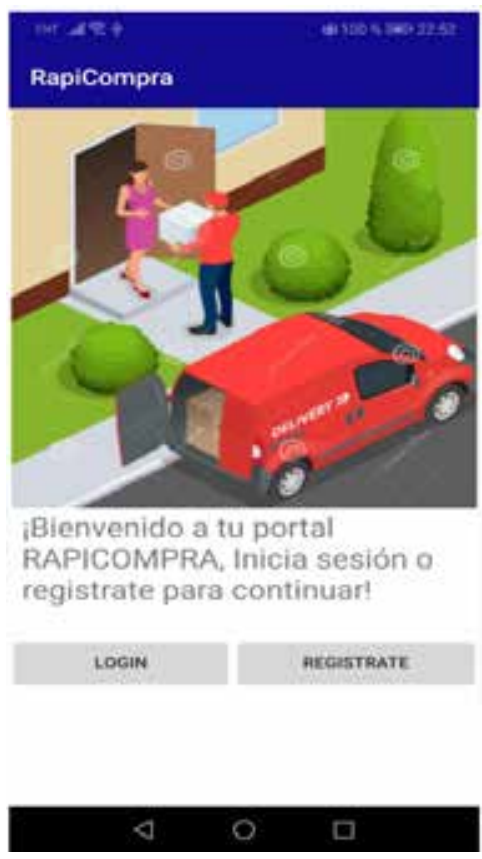

Figura 8. Pantalla de bienvenida

Fuente: elaboración propia

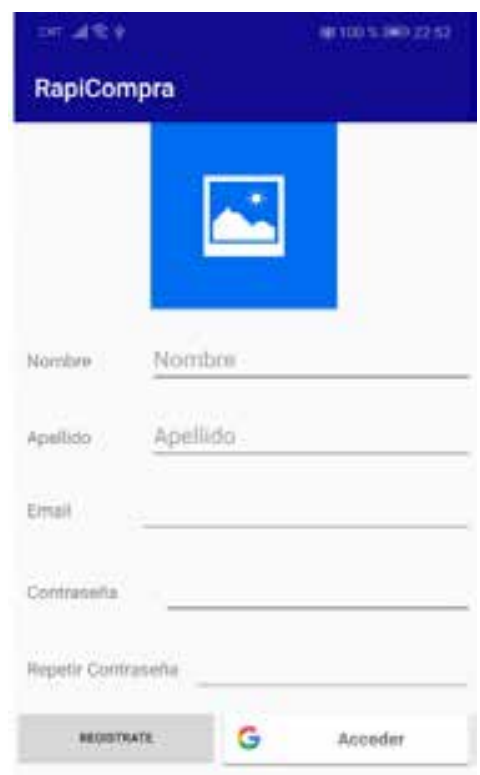

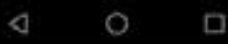

Figura 9. Pantalla de registro

Fuente: elaboración propia 


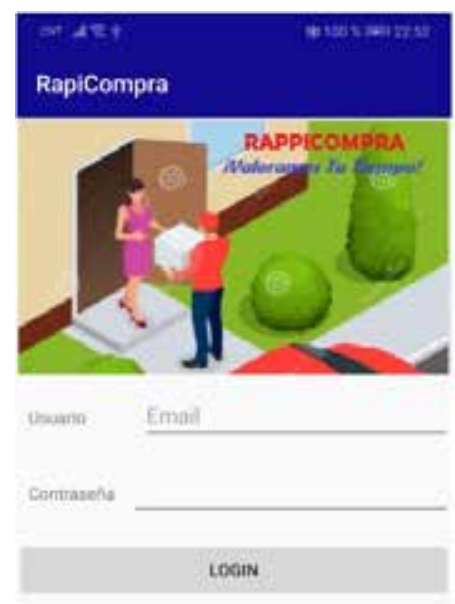

\section{$40 \quad \square$}

Figura 10. Pantalla de login

Fuente: elaboración propia

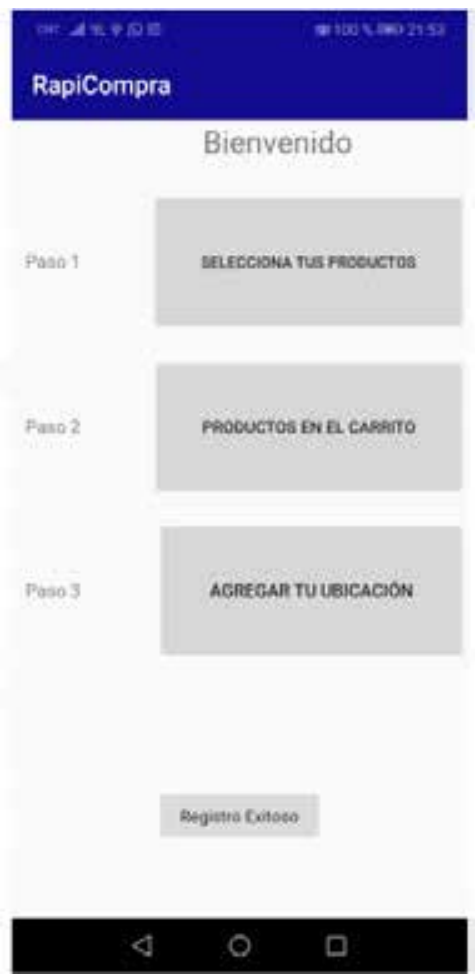

Figura 11. Pantalla de menú

Fuente: elaboración propia 


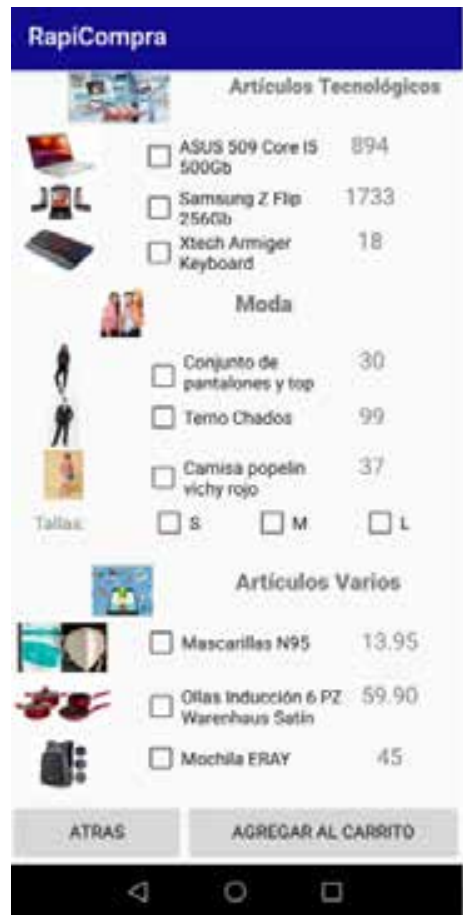

Figura 12. Pantalla de lista de productos

Fuente: elaboración propia

Una vez realizado su pedido se reflejará como se ve en la Figura 13.

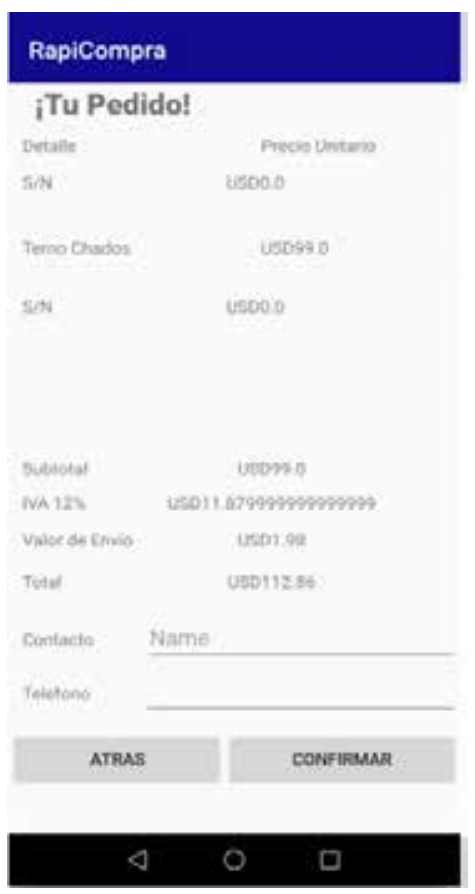

Figura 13. Pantalla de lista de pedidos

Fuente: elaboración propia 
Y en consiguiente podrá especificar su ubicación para recibir su pedido (Figura 14 y 15).

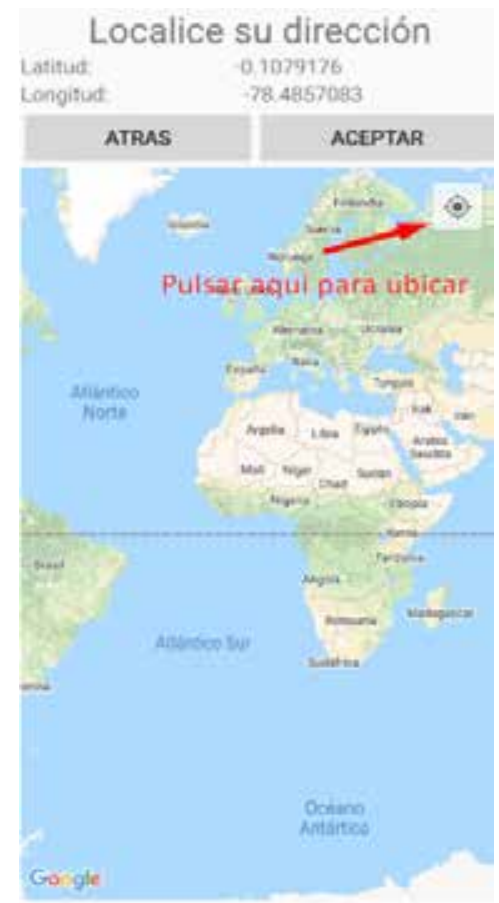

Figura 14. Pantalla de lista de Georreferenciación

Fuente: elaboración propia

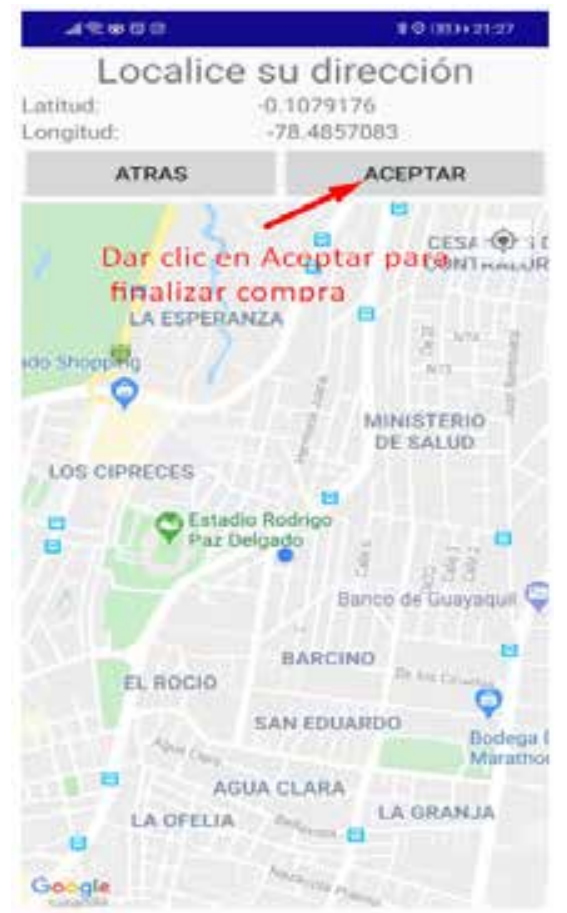

Figura 15. Pantalla con la ubicación de usuario

Fuente: elaboración propia 


\section{Conclusiones}

Una vez finalizado el proyecto se agregó una capa extra de seguridad usando la autentificación de Google, ya que el usuario podría activar el doble factor de autentificación previniendo una suplantación de identidad.

El uso de la API de mapas y geolocalización fue indispensable con el fin de tener una ubicación exacta para evitar errores de direcciones o mal entendidos, inclusive a nivel de usuario el tiempo que demoraría ingresar de forma manual la dirección versus un solo toque para localizar su ubicación, de esta manera el repartidor contará con el punto exacto de entrega.

Esta aplicación brindará la posibilidad a negocios del segmento SMB subir sus productos y ofrecerlo a su público de una manera rápida, visualmente atractiva y con una versatilidad de compra incomparable.

La gran ventaja de una aplicación móvil es que se puede compartir los requerimientos de cómputo con el dispositivo del cliente, ya que la aplicación se ejecutará $100 \%$ y usará recursos del celular y el computo adicional que requerimos es únicamente para almacenar y administrar la información ingresada en la aplicación, lo que en un ambiente $100 \%$ web tendríamos que dimensionar el computo de uso de la aplicación por cada usuario. 


\section{Referencias}

Android. (2019). Cómo agregar mapas. https://developer.android.com/training/maps

Android. (2019). Cómo mostrar la dirección de una ubicación. https://developer.android.com/training/location/ display-address?hl=es

Android. (2020). Cómo solicitar permisos de la app. https://developer.android.com/training/permissions/requesting?hl=es-419

Android. (2020). Cómo recordar y autenticar usuarios. https://developer.android.com/training/id-auth

Android, A. (2016). SQLite en Android: creación y acceso base de datos e inserción de registros. Academia Android. https://academiaandroid.com/sqlite-android-creacion-acceso-base-datos-insercion/

Basi. (2019). How to solve "Key was created with errors". StackOverFlow. https://stackoverflow.com/questions/56215400/how-to-solve-key-was-created-with-errors

Caldito, L. A. (2005). Medición de las actitudes de los internautas respecto a la compra on-line. Segmentación en base a actitudes y caracterización de los segmentos identificados. Economic Analysis Working Papers (2002-2010). Atlantic Review of Economics (2011-2016), 4, 1-26.

Contreras, C. (2018). Así nació Uber Eats, la revolución global del servicio de comida a domicilio. estrategiaynegocios.net. https://www.estrategiaynegocios.net/empresasymanagement/1235250-330/as\%C3\%AD-naci\%C3\%B3-uber-eats-la-revoluci\%C3\%B3n-global-del-servicio-de-comida-a

Dinu, G., \& Dinu, L. (2014). Using Internet as a commercial tool: A case study of e-commerce in Resita. Procedia Engineering, 69, 469-476. http://doi.org/10.1016/j.proeng.2014.03.014

Kreibich, J. (2010). Using SQLite. "O’Reilly Media, Inc”.

Li, F., \& Li, Y. (2011). Usability evaluation of e-commerce on B2C websites in China. Procedia Engineering, 15, 5299-5304.

Maldonado, E. J. (2016). ¿Cómo enviar datos entre activities? StackOverflow. https://es.stackoverflow.com/ questions/36902/como-enviar-datos-entre-activities

Scrum. (2020). Scrum. https://www.scrum.org/open-assessments/scrum-developer-open

Slotnisky, D. (2016). Transformación digital: cómo las empresas y los profesionales deben adaptarse a esta revolución. Digital House. Coding School. 